\title{
Monitored anesthesia care (MAC) sedation: clinical utility of fospropofol
}

This article was published in the following Dove Press journal:

Therapeutics and Clinical Risk Management

7 December 2009

Number of times this article has been viewed

\author{
Eric A Harris \\ David A Lubarsky \\ Keith A Candiotti \\ Department of Anesthesiology, \\ Perioperative Management, and Pain \\ Medicine, University of Miami/Miller \\ School of Medicine
}

\begin{abstract}
Fospropofol, a phosphorylated prodrug version of the popular induction agent propofol, is hydrolyzed in vivo to release active propofol, formaldehyde, and phosphate. Pharmacodynamic studies show fospropofol provides clinically useful sedation and EEG/bispectral index suppression while causing significantly less respiratory depression than propofol. Pain at the injection site, a common complaint with propofol, was not reported with fospropofol; the major patient complaint was transitory perianal itching during the drug's administration. Although many clinicians believe fospropofol can safely be given by a registered nurse, the FDA mandated that fospropofol, like propofol, must be used only in the presence of a trained anesthesia provider.

Keywords: fospropofol, propofol, monitored anesthesia care
\end{abstract}

\section{Introduction}

The concept of moderate sedation and analgesia, introduced to replace the more arcane term conscious sedation, has been generally accepted in the anesthesia community as an appropriate target for sedation by nonanesthesiologists. Moderate sedation as defined by the American Society of Anesthesiologists (ASA) requires that the patient be arousable to verbal commands or light tactile stimulation. A patent airway, as well as stable cardiac and respiratory functions, are maintained throughout the period of sedation (Table 1). ${ }^{1}$ Moderate sedation is not synonymous with monitored anesthesia care (MAC); the former can be administered by anyone capable of giving the medications and monitoring the patient, ${ }^{2}$ while MAC must be performed under the medical direction of an anesthesiologist. The scope of MAC is significantly wider, including the necessity of a preoperative evaluation, an anesthesiologist's personal participation or medical direction of the entire plan of care, and the ability to rescue a patient from unintended deep sedation or to intentionally provide deep sedation or general anesthesia if clinically warranted.

There is a common armamentarium of drugs shared between providers of moderate sedation and MAC, all given with the intent of maximizing anxiolysis and amnesia while maintaining a verbal patient. The ability of the patient to speak and understand is useful not only as a monitor of sedation depth and cardiorespiratory function but is also necessary to offer reassurance and communicate to the patient when active cooperation is required during the procedure (eg, breath holding). ${ }^{3}$ Hypnotic agents may be employed during MAC to bring the patient to a level of deep anesthesia/analgesia. Propofol, a shortacting anesthetic agent that is rapidly titratable, is currently the premier agent chosen to achieve this purpose. Qualities such as a quick recovery time (even after a prolonged
Correspondence: David A Lubarsky Emanuel M Papper Professor and Chair, Department of Anesthesiology, Perioperative Management, and Pain Medicine, University of Miami/Miller School of Medicine, I6II NW I2th Ave, Miami, FL 33I36, USA

Email dlubarsky@med.miami.edu 
Table I Definitions of clinical states of sedation as proposed by the American society of anesthesiologist's task force on sedation and analgesia by non-anesthesiologists

\begin{tabular}{ll}
\hline Sedation level & Characteristics \\
\hline Minimal sedation/anxiolysis & A drug-induced state during which patients respond normally to verbal commands. \\
Cognitive function and coordination may be impaired. \\
Ventilatory and cardiovascular functions are unaffected. \\
Moderate sedation/analgesia & A drug-induced depression of consciousness during which patients respond purposefully to verbal commands, \\
either alone or accompanied by light tactile stimulation. \\
No interventions are required to maintain a patent airway and spontaneous ventilation is adequate. \\
Cardiovascular function is usually maintained. \\
A drug-induced depression of consciousness during which patients cannot be easily aroused but respond \\
purposefully following repeated or painful stimulation. \\
Ability to independently maintain ventilatory function may be impaired. \\
Patients may require assistance in maintaining a patent airway and spontaneous ventilation may be inadequate. \\
Cardiovascular function is usually maintained. \\
A drug-induced loss of consciousness during which patients are not arousable, even by painful stimulation. \\
Ability to independently maintain ventilatory function is often impaired. \\
Patients often require assistance in maintaining a patent airway and positive pressure ventilation may be required \\
because of depressed spontaneous ventilation or drug-induced depression of neuromuscular function. \\
Cardiovascular function may be impaired.
\end{tabular}

Reproduced with permission from Practice guidelines for sedation and analgesia by non-anesthesiologists. An updated report by the American Society of Anesthesiologists Task Force on sedation and analgesia by non-anesthesiologists. Anesthesiology. 2002;96:1004-1017.' Copyright $\odot 2002$ Wolters Kluwer Health.

infusion) and the fact that the drug is not associated with nausea or emesis have further augmented its popularity.

Propofol was first introduced into clinical practice in 1986 by AstraZeneca under the trade name Diprivan ${ }^{\circledR}$ (a shortened version of DI-isoPRopyl IV ANesthetic.) It was marketed as an agent for the induction and maintenance of general anesthesia as part of a balanced technique, as well as the short term ( $<72$ hours) sedation of mechanically ventilated adults in the intensive care setting. Its popularity and safety profile resulted in its adoption in many sites outside of the operating room and ICU, and it soon became the drug of choice for providing sedation to patients undergoing minimally invasive and uncomfortable procedures such as bronchoscopy, transesophageal echocardiography, and colonoscopy. ${ }^{4}$ As such, it supplanted the standard regimens of intravenous benzodiazepine/narcotic combinations and the DTP cocktail (meperidine, promethazine, and chlorpromazine). ${ }^{5}$ Patients reported a high degree of satisfaction with propofol and regained consciousness quickly, thereby facilitating their discharge and speeding flow through a busy medical site. ${ }^{6}$ Although economic savings were garnered through reduced PACU stays, the necessity of trained personnel to deliver propofol added another layer of cost to the procedure. As per the package insert: "DIPRIVAN $2 \%$ should be given by those trained in anesthesia (or, where appropriate, doctors trained in the care of patients in intensive care). Patients should be constantly monitored and facilities for maintenance of a patent airway, artificial ventilation and oxygen enrichment and other resuscitative facilities should be readily available at all times. DIPRIVAN 2\% should not be administered by the person conducting the diagnostic or surgical procedure." In the case of colonoscopies, gastroenterologists estimate that the need for an anesthesiology provider adds between US $\$ 250$ and US $\$ 400$ to the cost of every procedure. ${ }^{8}$ This has galvanized many physicians, especially gastroenterologists, to petition the FDA to revise the requirement for a practitioner trained in anesthesia. As evidence, they cite several large studies ${ }^{9-12}$ that document the safety of $\mathrm{RN}$-administered propofol in the absence of an anesthesiologist. The ASA continues to support the current safety recommendations. ${ }^{13}$

Despite its widespread clinical use, propofol is not a drug that is free of unwanted side effects. Perhaps the most ubiquitous is pain at the injection site, ${ }^{14}$ a phenomenon that is unreliably reduced by the addition of lidocaine to the propofol solution prior to injection or by the injection of lidocaine into the vein prior to propofol. (The only technique shown to reliably reduce pain on injection in a majority (60\%) of patients is to apply a tourniquet to the proximal arm and administer lidocaine $0.5 \mathrm{mg} /$ kg 30 to 120 seconds prior to the propofol. $)^{15}$ Propofol infusion syndrome, a rare but reported condition, includes severe metabolic acidosis, rhabdomyolysis, renal failure, and cardiac failure in association with a prolonged propofol infusion, critical illness, and the concurrent administration of catecholamines and steroids. ${ }^{16}$ The lipid emulsion formula introduces another set of concerns including the need for absolute sterility when handling the drug, the relatively short window of usage (6 hours) when 
the vial is opened, and the hypertriglyceridemia seen in patients receiving propofol infusion in the ICU setting. ${ }^{17-19}$ Finally, propofol itself has a remarkably narrow therapeutic window; even in trained hands, the dose curve bridging moderate sedation to general anesthesia may be unexpectedly steep. In susceptible patients, propofol is known to cause dose-dependent hemodynamic ${ }^{20}$ and respiratory ${ }^{21}$ depression and possibly loss of airway protective reflexes ${ }^{22}$ in doses commonly used for mild to moderate sedation.

The stage was therefore set to develop a milder form of propofol - one with less pronounced cardiorespiratory depression, preferably delivered in an aqueous form to eliminate the problems associated with the lipid emulsion. Investigators had proven that hydrophobic drugs could be made water-soluble by the addition of a large hydrophilic group, typically a phosphate monoester or a hemisuccinate, to create a prodrug. The hydrophilic addition was then enzymatically cleaved in vivo releasing the active drug. This approach has been used successfully with a variety of drug classes including antibiotics and steroids, ${ }^{23}$ and more recently in the development of the anticonvulsant drug phosphenytoin. Initial animal studies were performed by annexing propofol with a variety of water-soluble side groups. ${ }^{24-30}$ Researchers reported success in the formulation of propofol phosphate, a water-soluble prodrug which reliably produced sedation in small mammals typical of that seen with propofol injection. ${ }^{31}$ This was followed by the development of Aquavan ${ }^{\circledR}$ (Guilford Pharmaceuticals, Baltimore, MD), initially referred to as GPI 15715. Chemically this water-soluble prodrug undergoes hydrolysis by alkaline phosphatase (predominantly at the endothelial cell surface) to release the active metabolite propofol, formaldehyde, and phosphate (Figure 1.) The liberated formaldehyde is rapidly converted to formate. Sedation and anesthesia are reliably produced among animals ${ }^{32}$ as well as human ${ }^{33}$ subjects.

\section{Pharmacokinetics}

The pharmacokinetics of fospropofol have been extensively studied in both laboratory animals as well as humans, and details have been incorporated into a web based simulation comparing fospropofol to propofol. ${ }^{35}$ In humans, a dual compartment model for the central distribution of the drugs was devised ${ }^{33,34,36}$ where the concentration of fospropofol in the central compartment was a function of the injected dose (D), and the concentration of propofol in the central compartment was a function of its conversion from fospropofol calculated in mass per time as $\mathrm{F} \times \mathrm{k}_{\text {met }} \times \mathrm{C}_{\mathrm{GPI}} \times \mathrm{V}_{\mathrm{CGPI}}$, where $\mathrm{F}$ equals the fraction of the dose of fospropofol that is metabolized to propofol, $\mathrm{k}_{\text {met }}$ is the elimination rate constant of the prodrug, $\mathrm{C}_{\mathrm{GPI}}$ is its plasma concentration, and $\mathrm{V}_{\mathrm{CGPI}}$ is the volume of distribution of fospropofol in the central compartment. $\mathrm{C}_{\mathrm{GPI}}$ is measured directly, $\mathrm{V}_{\mathrm{CGPI}}$ and $\mathrm{k}_{\text {met }}$ are estimated, and $\mathrm{F}$ is calculated using the molecular weights of fospropofol and propofol (332 and 178, respectively) assuming a complete conversion. The data also implicated the presence of peripheral compartments for both drugs, and nonlinear regression suggested the best fit for the data relied upon the presence of two peripheral compartments for each of the drugs, with transfer rate constants between the central and peripheral spaces designated as $\mathrm{k}_{12}$ and $\mathrm{k}_{21}, \mathrm{k}_{13}$ and $\mathrm{k}_{31}$, etc. (Figure 2).

Further examination of the pharmacodynamics of fospropofol revealed a biphasic elimination curve for the parent
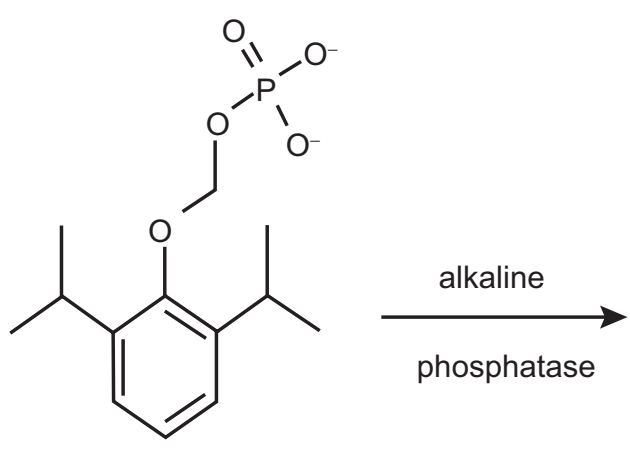

AQUAVAN $^{\circledR}$
Injection

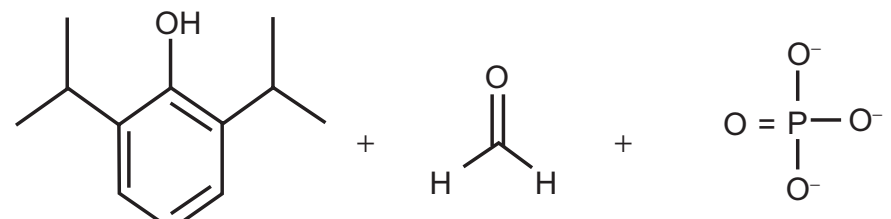

Propofol
Phosphate

Figure I The conversion of fospropofol into its metabolites. Reproduced with permission from Gibiansky E, Struys M, Gibiansky L, Vanluchene A, et al. Aquavan ${ }^{\circledR}$ injection, a water-soluble prodrug of propofol, as a bolus injection: a phase I dose-escalation comparison with Diprivan ${ }^{\circledR}$ (Part I - Pharmacokinetics). Anesthesiology. 2005; I03:7 I8-729. ${ }^{34}$ Copyright (c) 2002 Wolters Kluwer Health. 


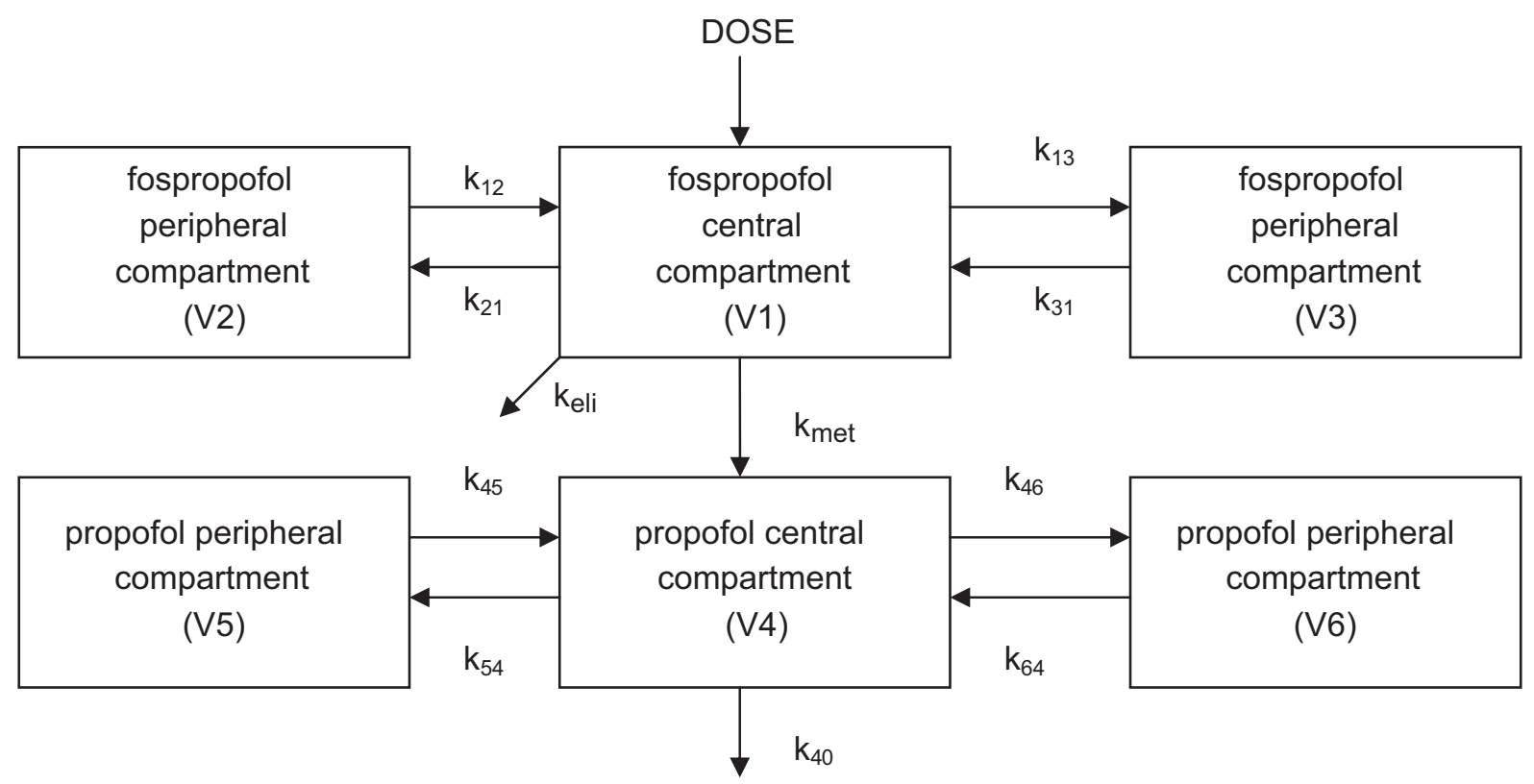

Figure 2 Some investigators suggest that fospropofol exists in a single peripheral compartment, thereby resulting in a 5 -compartment model. Others ${ }^{33}$ postulate a dual peripheral compartment for fospropofol, suggesting a 6-compartment model (shown).

drug, with a steep initial decline (representing fast elimination and redistribution within a small volume of distribution) followed by a slower second phase decline of secondary elimination and conversion. The gender of the volunteer was found to have no effect upon the pharmacokinetic profile. ${ }^{34}$ The liberated propofol, as expected, displayed typical lipophilic pharmacodynamics with large volumes of distribution. However, propofol derived from the parent prodrug showed significant differences in pharmacodynamic properties from Diprivan $^{\circledR}$, namely a larger volume of distribution, lower peak plasma concentrations, and a shorter half-life due to a more rapid clearance. These differences were initially attributed to differences in sampling procedures and study design. It was later realized that an error in the assay ${ }^{37}$ invalidated all of the quantitative pharmacokinetic data related to fospropofol. In all of the studies previously referenced samples of blood were collected in tubes containing a powdered form of sodium orthovanadate (SOV) to inhibit the alkaline phosphatase enzyme and therefore preclude the further conversion of fospropofol into propofol. Careful examination, however, later revealed incomplete dissolution of the powder resulting in various concentrations of SOV, thereby affecting plasma $\mathrm{pH}$ and in some instances causing hemolysis. Because these factors were neither known nor controlled at the time of the studies, all data derived relating to the propofol concentrations could therefore be inaccurate, and is therefore now considered invalid. However the quantitative data relating to fospropofol itself is legitimate, as it was not affected by the assay. Repeated assays using liquid SOV, which would preclude this problem, have been suggested, but at the time of this writing they have yet to be published.

Urine samples have also been studied to test for the excretion of unchanged fospropofol. Fewer than $5 \%$ of the samples revealed the presence of the compound in the urine, and the majority of those were from volunteers receiving fospropofol in the higher dose range. From these data, the fraction of unchanged fospropofol excreted in the urine is estimated at $0.02 \%{ }^{34}$

When fospropofol is converted to its active metabolite propofol, formate is released from the parent compound. In previous research formate, in high concentrations, has been shown to result in acidosis, ketonemia and acetonuria, respiratory compromise, and blindness. ${ }^{38}$ In controlled studies it was demonstrated that no significant difference in intravenous formate levels existed between patients receiving fospropofol or Diprivan ${ }^{\circledR}$. Furthermore, the level of intravenous formate was not found to vary with increasing doses of either of the induction agents. ${ }^{34}$

\section{Pharmacodynamics}

The pharmacodynamic properties of fospropofol have been studied using both noncompressed EEG evaluation ${ }^{39}$ as well as bispectral index (BIS) monitoring. Initial dose escalation studies were performed on nine healthy male volunteers 
divided into 3 groups of 3 volunteers. ${ }^{33}$ Each group received a fospropofol infusion over a 10-minute period, with the first group receiving a total dose of $290 \mathrm{mg}$ each, the second group receiving $580 \mathrm{mg}$ each, and the final group receiving $1160 \mathrm{mg}$ each. The volunteers were tested for loss of consciousness (LOC) as defined in this study by the absence of a response to a loud verbal command. If LOC was documented, the patient was further tested for a corneal reflex response, defined in this study as being a physical response to having a wisp of cotton rubbed across the cornea.

Among the group receiving $290 \mathrm{mg}$ of fospropofol, no LOC was documented. One patient in this group reported an unpleasant sensation of tingling and burning in the anal and genital area lasting approximately 5 minutes which resolved without therapy. Among the 3 patients receiving $580 \mathrm{mg}, 1$ experienced LOC 12 minutes after the initiation of the infusion and return to consciousness (ROC) was noted 22 minutes after the start of the infusion. Blood concentrations of propofol were obtained corresponding to LOC and ROC, but the aforementioned error in propofol analysis has invalidated the accuracy of these measurements. Amongst the highest dose group all 3 patients displayed LOC $9 \pm 3$ minutes after the start of the infusion. ROC occurred $24 \pm 2$ minutes after fospropofol infusion was initiated. In this group one patient again complained of a burning sensation in the anogenital region, spontaneously resolving after 2 minutes. Among the 4 patients who experienced LOC, the administered dose of fospropofol was $870 \pm 237 \mathrm{mg}$ (mean \pm SD). The corneal reflex was lost in only 1 patient, a member of the $1160 \mathrm{mg}$ group.

All 9 subjects were also evaluated using the Observer's Assessment of Alertness/Sedation (OAA/S) Scale. ${ }^{40}$ The scale was evaluated at 2, 5, 10, 20, 60, 120, and 240 minutes after the conclusion of the infusion (for patients who experienced no LOC) or after ROC. Patients were graded on a scale from 1 (deep sleep) to 5 (completely alert). The low dose group achieved a score of 5 in $25 \pm 5$ minutes, the middle dose group at $63 \pm 49$ minutes, and the high dose group at $112 \pm 72$ minutes.

The authors of the study also sought to measure the hemodynamic effects of fospropofol on the 9 volunteers. While one subject in the $580 \mathrm{mg}$ group displayed an elevation in systolic blood pressure throughout the entire study period, the remaining subjects all showed a decrease in both systolic and diastolic pressures in the range of $20 \%$ to $25 \%$. (In order of ascending dose, the values were $\mathrm{S}:-18 \% \pm 7 \%$, D: $-13 \% \pm 9 \%$ for Group 1; S: $-18 \% \pm 15 \%$, D: $-29 \% \pm 7 \%$ for Group 2; S: $-25 \% \pm 8 \%$, D: $-28 \% \pm 9 \%$ for Group 3).
Blood pressure values reached their nadir at $20 \pm 8$ minutes after the beginning of fospropofol infusion and returned to baseline approximately 60 minutes after the start of the infusion. All subjects showed an increase in heart rate (in order of ascending dose, $+36 \% \pm 17 \%,+32 \% \pm 4 \%$, and $+52 \% \pm 35 \%$ ) including 1 volunteer in Group 3 who had an elevation of heart rate from 43 to 83 beats per minute. Heart rate reached its maximum value at $12 \pm 8$ minutes after the initiation of fospropofol infusion and returned to baseline at approximately 30 minutes after the start of the infusion.

In addition, respiratory and metabolic parameters were also measured. In all 9 subjects oxygen saturation dropped to a minimum value of $94.6 \% \pm 1.6 \%$, reached $15 \pm 3$ minutes after the beginning of the fospropofol infusion. All 3 patients in the $1160 \mathrm{mg}$ dose group required insufflation of oxygen via a nasal cannula secondary to an oxygen saturation via pulse oximetry of less than $93 \%$. Apnea was not observed in any of the subjects. An arterial blood sample drawn from each volunteer at the end of the infusion revealed a dose dependent rise in $\mathrm{PaCO}_{2}$ in the 3 ascending dosage categories to $38.2 \pm 2.7,42.9 \pm 0.9$, and $47.1 \pm 4.8 \mathrm{~mm} \mathrm{Hg}$ respectively. Body temperature remained constant in all subjects at $36.2 \pm 0.4^{\circ} \mathrm{C}$.

It is difficult to compare these physiologic results to what one would find in patients who received an equipotent dose of propofol in lipid emulsion. Prior studies involved infusion of propofol over longer time intervals (Forrest et al delivered $500 \mathrm{mg}$ of propofol over 30 minutes and reported a drop in systolic blood pressure of $22 \%$, a diastolic drop of $28 \%$, and an increase in pulse rate of $12 \%) .{ }^{41}$ Other authors report a more modest drop of systolic BP of $15 \%,{ }^{42}$ but the achieved concentration may not correlate with the fospropofol study.

The same authors expanded upon their study protocol the following year by studying pharmacodynamics not only in terms of clinical signs of sedation, but also by collecting EEG and BIS data. ${ }^{36}$ Again 9 male volunteers were recruited in the study. In this protocol, each volunteer received a propofol lipid emulsion infusion over 60 minutes with the dose adjusted to obtain a specific plasma propofol concentration. For the first 20 minutes of the infusion, the target plasma concentration was $5 \mu \mathrm{g} / \mathrm{mL}$; this was reduced to $3 \mu \mathrm{g} / \mathrm{mL}$ for the next 20 minutes and $1.5 \mu \mathrm{g} / \mathrm{mL}$ for the final 20 minutes. The rationale behind the varying target plasma concentrations was to more accurately measure clinical pharmacodynamic effects. ${ }^{43}$ A constant infusion, if set too low, may not produce the desired clinical signs. Alternately, if it is set too high the clinical signs may occur 
in such rapid succession that it is difficult or impossible to correlate the pharmacodynamic effect with the plasma concentration of the drug. Patients were monitored for physiologic signs as well as signs of sedation identical to the monitoring described in the authors' previous study. In addition, EEG monitoring was started 30 minutes prior to the beginning of the infusion and continued until the patient was alert as determined by the OAA/S Scale. BIS monitoring via an Aspect A1000 ${ }^{\circledR}$ monitor (Aspect Medical Systems, Norwood MA) was also performed during this interval.

The same subjects were brought back at least 14 days later to repeat the study; this time, however, they received a continuous 60-minute infusion of fospropofol. The aim of the study was to adjust the drug concentration to replicate the plasma propofol concentration in the identical 3 intervals: 20 minutes at $5 \mu \mathrm{g} / \mathrm{mL}, 20$ minutes at $3 \mu \mathrm{g} / \mathrm{mL}$, and 20 minutes at $1.5 \mu \mathrm{g} / \mathrm{mL}$. However, due to the previously discussed problem with SOV, the measured propofol concentrations may have been incorrect, falsely suggesting an equivalence in dosage between propofol lipid solution and fospropofol when such an equivalence didn't exist.

The EEG results for the propofol group showed initial high activity in the $\alpha$ band with a median frequency (MEF) of 9 to $10 \mathrm{~Hz}$. Within 5 minutes of beginning the infusion $\beta$ activity began to appear with a concomitant drop in the MEF to $2.5 \mathrm{~Hz}$. This was followed by a shift to the slower $\theta$ and $\delta$ wavelengths. As the propofol plasma concentration was dropped during the second 20 minute interval the MEF was noted to increase to $3.5 \mathrm{~Hz}$, and it continued to trend towards baseline during the third 20 minute interval. No burst suppression was noted during the 1-hour infusion. Out of the 9 volunteers, 6 dropped their BIS from initial readings of approximately 90 to minimum values of 50 to 60 . One patient began with a baseline BIS reading of 75 , and the 2 remaining volunteers never dropped their BIS below 80. When the patients later received fospropofol the EEG showed a more rapid decrease in MEF, dropping quickly to $1 \mathrm{~Hz}$ and remaining less than $3 \mathrm{~Hz}$ throughout the duration of the infusion. $\beta$ activity was not noted as the patients progressed directly to the $\theta$ and $\delta$ wavelengths, although burst suppression was present in several volunteers. MEF did not return to baseline until approximately 30 minutes after the cessation of the infusion. For the fospropofol group the reduction in BIS was of a comparable amount, although the slope of the drop was steeper and the duration was prolonged versus the propofol group. Again, the equivalence of dosing cannot be relied upon.
Hemodynamic and respiratory parameters were similar between the two series, with blood pressure decreasing approximately $30 \%$ and heart rate increasing by approximately 40\%. Apnea was not observed in any subjects, but supplemental oxygen via nasal cannula (to treat $\mathrm{SpO}_{2}<93 \%$ ) was required for 6 of the 9 patients receiving propofol and all 9 patients receiving fospropofol. At 20 minutes after the start of infusion the $\mathrm{PaCO}_{2}$ was slightly higher in the group receiving fospropofol $(51.1 \pm 4.1 \mathrm{mmHg}$ vs $48.0 \pm$ $3.1 \mathrm{mmHg}$.) Inorganic phosphate remained within normal limits (2.5-4.5 mg/dL) for the propofol group but was slightly elevated at 20 minutes and 60 minutes after the start of the fospropofol infusion $(4.8 \pm 4.3$ and $4.7 \pm 0.6 \mathrm{mg} / \mathrm{dL}$, respectively). All subjects displayed normal inorganic phosphate levels within 24 hours. No increase in formate concentration was noted in either group.

Subjectively, 3 of the 9 subjects reported pain at the injection site during the propofol trial, beginning with the initiation of infusion and persisting for 4 to 5 minutes. In contrast, none of the volunteers reported localized pain during the fospropofol administration. However, all 9 volunteers reported itching, burning, and paresthesia in the anal and genital regions beginning 1 minute after the infusion had begun and resolving within 1 to 2 minutes. 2 of the subjects reported the severity as mild, 6 reported moderate symptoms, and 1 reported severe manifestations. Mild to moderate myoclonus was also noted in 3 of the 9 subjects, beginning 16 to 22 minutes after the start of the fospropofol infusion and lasting from 8 to 12 minutes before spontaneous resolution.

In 2005, a study was performed using burst suppression ratio and BIS monitoring to compare and contrast the pharmacodynamic profiles of propofol and fospropofol. ${ }^{44} \mathrm{By}$ using purely clinical data, they circumvented the SOV issue that had plagued prior studies. Thirty-six healthy volunteers were divided into 6 groups of 6 and given a bolus dose of fospropofol $(5,10,15,20,25$, or $30 \mathrm{mg} / \mathrm{kg}$ in Groups 1-6, respectively). The lowest BIS value $\left(\mathrm{BIS}_{\text {peak }}\right.$ ) as well as the time required to obtain this level of hypnosis $\left(T_{\text {BIS, peak }}\right)$ was recorded for each subject. Hemodynamic and respiratory parameters were also recorded. All subjects were then brought back one week later and received a continuous infusion of propofol (50 mg/minute) titrated to similar BIS peak $_{\text {as }}$ the prior week.

Among the 6 groups, only Groups 5 and 6 displayed a burst suppression ratio of greater than $10 \%$. Both fospropofol and propofol produced a dose dependent decrease in BIS $_{\text {peak }}$, although $\mathrm{T}_{\mathrm{BIS} \text {, peak }}$ was significantly shorter when patients received propofol rather than fospropofol. Loss of 
consciousness to verbal stimuli occurred with both drugs in Groups 3-6 at statistically similar BIS values and time measurements. It is interesting that both drugs displayed a statistically similar time until $\mathrm{LOC}_{\text {verbal, }}$, but thereafter propofol produced a $\mathrm{T}_{\text {BIS, peak }}$ more quickly than fospropofol. Duration of unconsciousness increased with increasing doses of both drugs, but fospropofol produced a significantly longer $\mathrm{LOC}_{\text {verbal }}$ than propofol among each cohort.

Hemodynamics were also studied for both drugs. Both drugs produced a transitory tachycardia $(25 \%-40 \%$ increase with fospropofol, 10\%-20\% with propofol) within the first minute after administration. All cases were transient and resolved spontaneously. It is not known whether the change in heart rate was due to a pharmacodynamic effect of the drugs or whether the patients were reacting to an uncomfortable sensation (one third of the propofol patients reported pain at the injection site, while all 36 fospropofol patients reported genital and perianal paresthesias). The majority of subjects who received fospropofol showed a biphasic profile in their MAP, with 30/36 showing a small initial increase in MAP (10-15 mmHg) within the first minute, followed by a smooth drop in MAP of $20 \%$ to $25 \%$ from baseline values. As with the tachycardia, the increase in MAP may be attributable to the uncomfortable paresthesias experienced by these patients. In contrast, propofol resulted in an insignificant initial rise in MAP, followed by a more rapid drop (compared to fospropofol) of $20 \%$ below baseline, consistent with prior studies. ${ }^{41,45}$

While respiratory depression was noted with both drugs, fospropofol (as opposed to propofol) resulted in no episodes of apnea among the lowest dosed group. Fospropofol did not result in apnea until the threshold of $15 \mathrm{mg} / \mathrm{kg}$ was reached. For Groups 3-6, the number of volunteers with apnea, as well as the duration of the apneic periods, was statistically increased in the propofol group compared to the fospropofol group.

Patients in both cohorts were also subject to a battery of laboratory exams. Hematologic, electrolyte, and serum chemistry panels showed no variation from baseline after administration of either drug. Formate concentration showed no increase above baseline even among volunteers receiving the highest dose of fospropofol. Levels of inorganic phosphate were elevated in patients receiving the highest dose of fospropofol, yet they spontaneously returned to a normal range prior to reaching toxic levels. Ionized calcium remained unchanged in both cohorts. Cholesterol, low-density lipoprotein cholesterol, and high-density lipoprotein cholesterol values remained stable in both groups, although triglyceride levels were elevated in the volunteers receiving the higher doses of propofol; maximum elevation was reached 10 minutes after the start of the infusion and all returned to normal thereafter. As expected, no increase in triglycerides was noted after the administration of fospropofol.

Fechner et al studied the efficacy of using a 2-hour infusion of fospropofol to induce a level of sedation that would theoretically be adequate for a minimally invasive procedure. ${ }^{46}$ Their study group of 12 volunteers received a target controlled infusion (TCI) of the drug set to result in a propofol blood level of $1.8 \mu \mathrm{g} / \mathrm{mL}$ during the first hour. Again, their calculations rested upon data rendered inaccurate by the use of SOV and can only be evaluated qualitatively. Their goal during the first hour was to achieve a modified OAA/S (MOAA/S $)^{47}$ score (Table 2 ) of 2 or 3 within 60 minutes. If any patient was outside of this range the dose would be adjusted either upward or downward during the second hour in a further attempt to reach the target. Physiologic monitoring including BIS was performed throughout the study.

After 1 hour 9 of the 12 volunteers required an upward titration in their fospropofol infusions to achieve a satisfactory MOAA/S score. No patients required a downward titration due to oversedation. During the first hour the median MOAA/S score was 4 and the mean BIS was $72 \pm 12$. During the second hour the MOAAS score dropped to a median of 3 with a corresponding decrease in the BIS to a mean value of $61 \pm 11$. Changes in systolic and diastolic blood pressure, heart rate, and oxygen saturation were consistent with previously published data. 11 of the 12 volunteers complained of genital and perianal paresthesias beginning 1 minute after the start of the infusion and resolving 2 minutes thereafter. Four volunteers rated the sensation as mild and 7 reported it as moderate.

Although the pharmacokinetics of the study were flawed there are some salient points that were not apparent from the previously described fospropofol bolus studies. Among

Table 2 The Modified Observer's Assessment of Alertness/ Sedation (MOAA/S) scale

\begin{tabular}{ll}
\hline MOAA/S score & Clinical manifestations of sedation \\
\hline 5 & Responds readily to name spoken in normal tone. \\
4 & Lethargic response to name spoken in normal tone. \\
3 & $\begin{array}{l}\text { Responds only after name is called loudly and/or } \\
\text { repeatedly. }\end{array}$ \\
2 & Responds only after a painful trapezius squeeze. \\
\hline & Does not respond to a painful trapezius squeeze. \\
\hline
\end{tabular}


the nine patients who required an upward titration in their infusion, an increased level of sedation was reached at an average time of 3 minutes after the infusion rate was adjusted. This level of sedation was maintained during the remainder of the study. This suggests that fospropofol, like propofol, can be rapidly titrated in clinical practice to achieve a desired level of sedation. Recovery time after a 2-hour infusion was significantly higher for the fospropofol: after the 2 hour infusion the mean recovery time to a MOAA/S score of 5 was 18 minutes, approximately 10 minutes longer than the recovery time for propofol. ${ }^{48}$

\section{Clinical studies}

Clinical studies with fospropofol have been conducted among patients receiving bronchoscopies or colonoscopies. In the former, the vast majority of the 500,000 flexible bronchoscopies performed annually in the United States ${ }^{49}$ are performed under some type of intravenous sedation. ${ }^{50,51}$ While some authors have questioned the need for sedation during a flexible bronchoscopy, ${ }^{52}$ the majority of clinicians use a combination of a benzodiazepine (to provide amnesia) and an opiod (to provide both analgesia and an antitussive effect).$^{53}$ One multicenter study has suggested that fospropofol may be a good alternative. ${ }^{54}$ In this study, patients were randomized to receive either $2 \mathrm{mg} / \mathrm{kg}$ (nontherapeutic dose) or $6.5 \mathrm{mg} / \mathrm{kg}$ fospropofol prior to flexible bronchoscopy. All patients also received fentanyl $50 \mu \mathrm{g}$ iv and topical lidocaine spray. Fospropofol was redosed every 4 minutes, up to three times, if the MOAA/S score was 5 . The primary end point of the study was successful sedation (defined as three consecutive MOAA/S scores $\leq 4$ ) and successful treatment (defined as the ability to complete the bronchoscopy without the use of additional sedatives or assisted ventilation). Secondary end points included patient satisfaction with the procedure (defined as the willingness to undergo a repeat bronchoscopy with fospropofol), amnesia for the event, and time to recovery from sedation.

The higher dose group fared significantly better in achieving both primary end points. Among the $6.5 \mathrm{mg} / \mathrm{kg}$ group sedation success was $88.7 \%$ vs $27.5 \%$ for the $2 \mathrm{mg} / \mathrm{kg}$ group $(P<0.001)$; treatment success also heavily favored the higher dose group $(91.3 \%$ vs $41.2 \%, P<0.001)$. The majority $(56 \%)$ of the $6.5 \mathrm{mg} / \mathrm{kg}$ group required no supplemental doses of fospropofol, and only $8 \%$ required the addition of a benzodiazepine (compared to $7 \%$ and $58.8 \%$, respectively, in the $2 \mathrm{mg} / \mathrm{kg}$ group). The time interval until the patient was adequately sedated was 4 minutes in the high dose group vs 18 minutes in the low dose group. Secondary endpoints also favored the higher dose: among the $6.5 \mathrm{mg} / \mathrm{kg}$ group, $94.6 \%$ would be willing to repeat the procedure with fospropofol and $83.3 \%$ did not have any recall of the event (vs $78.2 \%$ and $55.4 \%$, respectively, among the $2 \mathrm{mg} / \mathrm{kg}$ group, $P<0.001$ for both). Readiness for hospital discharge was slightly prolonged in the higher dose group (8.5 vs 8.0 minutes) although the difference was statistically insignificant. (This should be contrasted with the 20- to 120-minute range for discharge readiness reported after the use of a benzodiazepine/opiod combination). ${ }^{55,56}$ Adverse events reported in both groups included pruritis, hypotension, and oxygen saturation below $92 \%$. All were rated by the patients and bronchoscopists as being mild to moderate and resolved either spontaneously or with minor intervention (eg, increased oxygen flow, chin lift, fluid bolus). The incidence of desaturation below $92 \%$ (15.4\% in the high dose group, $12.6 \%$ in the low dose group) was lower than prior published studies (24-32\%) ${ }^{57,58}$ using a benzodiazepine/opioid combination.

The number of colonoscopies performed annually far eclipses the number of bronchoscopies due in part to the former's use as a screening tool as well as a diagnostic procedure. As with bronchoscopies, sedation has become the standard of care during colonoscopies ${ }^{59}$ and has been shown to reduce the incidence of studies aborted prematurely due to patient intolerance. ${ }^{60,61}$ While agents such as benzodiazepines and opiods are frequently relied upon, gastroenterologists have become increasingly attracted to the use of propofol during colonoscopies. ${ }^{62}$ Despite the package insert warning that propofol should be "administered only by persons trained in the administration of general anesthesia and not involved in the conduct of the surgical/diagnostic procedure"7 gastroenterologists have been lobbying for this clause to be dropped, insisting that propofol can be safely administered by a registered nurse under the supervision of the physician performing the procedure. ${ }^{11,63-66}$ Both the American Society of Anesthesiologists and the American Association of Nurse Anesthetists have filed formal rebuttals arguing that the requested change is ill-advised. ${ }^{67}$ The small but genuine incidence of adverse effects, coupled with the reluctance of payors to compensate for the anesthesia component of routine colonoscopies ${ }^{68}$ has focused attention on fospropofol as being an all-purpose solution.

Clinical studies suggest that fospropofol may be as efficacious as propofol to provide rapid and safe sedation during routine outpatient colonoscopies. ${ }^{69}$ One study showed both drugs provided significant reduced time to discharge (and associated economic savings). In the time to complete 1 colonoscopy using midazolam and meperidine for sedation 
(71.1 minutes), the clinicians were able to complete 1.76 colonoscopies using propofol and 1.91 using fospropofol, resulting in an increased profit margin of around US\$67 per colonoscopy performed in a hospital outpatient setting, and US\$57 per procedure performed in an ambulatory surgical center. ${ }^{70}$ (These figures represent the profit for the gastroenterologist in the absence of an anesthesia provider. When such a provider is present and compensated, the average profits for the colonoscopy drop to US\$32 and US\$22, respectively). Savings attributed to rapid recovery were not analyzed from the perspectives of the patients (eg, less need for childcare) and society (eg, fewer days absent from work).

A common thread running throughout the gastrointestinal literature is that propofol (due to its lack of analgesic properties) is insufficient alone to provide moderate sedation necessary for a successful colonoscopy. ${ }^{71}$ The addition of a small dose of a benzodiazepine (which also has no analgesic properties) and/or an opiod (and possibly diphenhydramine as well $)^{72}$ has been shown to increase patient satisfaction, ${ }^{73}$ reduce the dose of propofol by up to $50 \%,{ }^{74}$ and reduce the time to discharge from the recovery area. ${ }^{75}$ This practice has extended to include fospropofol, as evidenced by a large study of patients receiving fospropofol for sedation during colonoscopies: all patients were premedicated with fentanyl $50 \mu \mathrm{g}$ before initiation of the sedation regimen. Patients were then randomized to receive either fospropofol $(2,5,6.5$, or $8 \mathrm{mg} / \mathrm{kg})$ or midazolam $(0.02 \mathrm{mg} / \mathrm{kg}){ }^{76}$ The goal was to maintain a MOAA/S score $\leq 4$; if necessary fospropofol was redosed every 4 minutes at one-quarter of the original dose (for the fospropofol group) or midazolam was redosed every 2 minutes at $1 \mathrm{mg}$ increments (for the benzodiazepine group). Results were similar to the bronchoscopy study referenced earlier; patients in the 6.5 and $8 \mathrm{mg} / \mathrm{kg}$ groups had statistically significant greater success in sedation and treatment success when compared to their counterparts in the 2 and $5 \mathrm{mg} / \mathrm{kg}$ cohort $(P<0.001)$. Patients in the $8 \mathrm{mg} / \mathrm{kg}$ dose group were much more likely to enter a state of deep sedation (defined as a MOAA/S score of 0 or 1) vs patients in the $6.5 \mathrm{mg} / \mathrm{kg}$ group ( $25 \%$ vs $4 \%$, respectively). Finally, patients in the $6.5 \mathrm{mg} / \mathrm{kg}$ group scored higher than any other group in measurements of patient satisfaction and willingness to repeat the procedure with the same method of sedation. No serious adverse effects were noted in any of the groups, and again the principle patient complaint was mild to moderate paresthesia.

\section{Conclusions}

Monitored anesthesia care provides a valuable bridge between moderate sedation, (which may be inadequate for a given procedure) and general anesthesia (which may be unnecessary). Under the direction of an anesthesiologist the patient can be both medically managed and safely sedated to allow for successful completion of the procedure. Fospropofol may prove to be a useful tool for the anesthesia provider, offering many of the benefits of propofol while eschewing several of the concomitant side effects. The most prevalent side effect of fospropofol, genital and perianal itching, has not interfered with the widespread clinical adoption of other phosphorylated prodrugs (eg, phosphenytoin) which share the same side effect profile. ${ }^{77}$

In mid-December 2008 the FDA approved fospropofol for use in monitored anesthesia care settings. ${ }^{78}$ Due to a series of corporate takeovers, fospropofol (GPI 15715; Aquavan $^{\circledR}$ ) will be marketed by the Eisai Corporation of North America under the trade name Lusedra ${ }^{\circledR}$. Like propofol, the FDA has mandated that Lusedra ${ }^{\circledR}$ be used only by persons trained in the administration of general anesthesia, and that all patients should be continuously monitored by persons not involved in the conduct of the procedure. ${ }^{79}$ The fact that fospropofol is not an induction agent has led some pulmonologists to feel it is safe to circumvent the requirement for trained anesthesia personnel during its administration. ${ }^{80}$ Other clinicians may join their chorus and petition the FDA for more liberal labeling. Some speculate that the FDA may proceed in a diametrically opposite direction, petitioning the DEA to classify Lusedra ${ }^{\circledR}$ as a controlled substance. ${ }^{81}$ We agree that while fospropofol is not an induction agent, the possibility exists that a patient may still proceed to an unintended depth of sedation. Given that risk, and the concomitant risk of aspiration and cardiopulmonary compromise, we believe that (as with propofol) the use of fospropofol should be limited to those clinicians trained in the practice of general anesthesia and rescue techniques. However the FDA proceeds, fospropofol should prove to be a useful adjunct for anesthesia providers administering monitored anesthesia care.

\section{Disclosures}

No outside funding or support was provided with respect to the authorship of this article, and the authors declare no conflicts of interest.

\section{References}

1. Practice guidelines for sedation and analgesia by non-anesthesiologists. An updated report by the American Society of Anesthesiologists Task Force on sedation and analgesia by non-anesthesiologists. Anesthesiology. 2002;96:1004-1017.

2. http://www.jointcommission.org/AccreditationPrograms/AmbulatoryCare/Standards/09_FAQs/PC/Moderate_Sedation_Medication.htm 
3. Hillier SC. Monitored anesthesia care. In: Barash PG, Cullen BF, Stoelting RK, eds. Clinical Anesthesia, 3rd ed. Philadelphia PA: Lippincott-Raven Publishers; 1997:1159-1171.

4. Kulling D, Rothenbuhler R, Inauen W. Safety of nonanesthetist sedation with propofol for outpatient colonoscopy and esophagogastroduodenoscopy. Endoscopy. 2003;35:679-682.

5. Snodgrass WR, Dodge WF. Lytic/'DTP' cocktail: time for rational and safe alternatives. Pediat Clin North Am. 1989;36(5):1285-1291.

6. Chen SC, Rex DK. Review article: registered nurse-administered propofol sedation for endoscopy. Aliment Pharmacol Ther. 2004;19: $147-155$.

7. Diprivan ${ }^{\circledR}$ [package insert]. AstraZeneca, February 2007.

8. Smith M. Furor surrounds Diprivan ${ }^{\circledR}$ use for colonoscopy. URL: http:// www.medpagetoday.com/Gastroenterology/ColonCancer/2398.

9. Cohen LB, Dubovsky AN, Aisenberg J, Miller KM. Propofol for endoscopic sedation: a protocol for safe and effective administration by the gastroenterologist. Gastrointest Endosc. 2003;58:725-732.

10. Heuss LT, Schnieper P, Drewe J, Pfimlin E, et al. Risk stratification and safe administration of propofol by registered nurses supervised by the gastroenterologist: a prospective observational study of more than 2000 cases. Gastrointest Endosc. 2003;57:664-671.

11. Rex DK, Overley CA, Walker J. Registered nurse-administered propofol sedation for upper endoscopy and colonoscopy: why? When? How? Rev Gastroenterol Disord. 2003;3:70-80.

12. Rex DK, Overley C, Kinser K, Coates M, et al. Safety of propofol administered by registered nurses with gastroenterologist supervision in 2000 endoscopic cases. Am J Gastroenterol. 2002;97:1159-1163.

13. Statement on safe use of propofol, approved by the ASA House of Delegates on October 27, 2004. URL: http://www.asahq.org/ publicationsAndServices/standards/37.pdf

14. Nakane M, Iwama $H$. A potential mechanism of propofol-induced pain on injection based on studies using nafamostat mesilate. Br J Anaesth. 1999;83:397-404.

15. Picard P, Tramer MR. Prevention of pain on injection with propofol: A quantitative systematic review. Anesth Analg. 2000;90:963-969.

16. Vasile B, Rasulo F, Candiani A, Latronico N. The pathophysiology of propofol infusion syndrome: a simple name for a complex syndrome. Intensive Care Med. 2003;29:1417-1425.

17. Albrecht S, Ihmsen H, Suchodolski K, Frenkel C, et al. Analgo-sedation in intensive care: A quantitative, EEG-based trial with propofol $1 \%$ and 2\%. Anaesthetist. 1999;48:794-2801.

18. Lindholm M. Critically ill patients and fat emulsions. Minerva Anestesiol. 1992;58:875-879.

19. McLeond G, Dick J, Wallis C, Patterson A. Propofol 2\% in critically ill patients: effects on lipids. Crit Care Med. 1997;25:1975-1981.

20. Smith I, White PF, Nathanson M. Propofol: An update on its clinical use. Anesthesiol. 1994;81:1005-1043.

21. Graber RG. Propofol in the endoscopy suite: an anesthesiologist's perspective. Gastrointest Endosc. 1999;49:803-806.

22. Sundman E, Witt H, Sandin R, Kuylenstierna R. Pharyngeal function and airway protection during subhypnotic concentrations of propofol, isoflurane, and sevoflurane: volunteers examined by pharyngeal videoradiography and simultaneous manometry. Anesthesiol. 2001;95: $1125-1132$.

23. Stella VJ, Charman WNA, Naringrekar VH. Prodrugs: do they have advantages in clinical practice? Drugs. 1985;29:455-473.

24. Trapani F, Latrofa A, Franco M, Lopedota A, et al. Inclusion complexation of propofol with 2-hydroxypropyl- $\beta$-cyclodextrin: physicochemical, nuclear magnetic resonance spectroscopic studies, and anesthetic properties in rats. J Pharm Sci. 1998;87:514-518.

25. Trapani F, Lopedota A, Franco M, Latrofa A, et al. Effect of 2-hydroxypropyl- $\beta$-cyclodextrin on aqueous solubility of the anaesthetic agent propofol (2,6-diisopropylphenol). Int J Pharm. 1996;139:215-218.

26. Bettschart-Wolfensberger R, Semder A, Alibhai H, Demuth D, et al. Cardiopulmonary side-effects and pharmacokinetics of an emulsion of propofol (Disoprivan ${ }^{\circledR}$ ) in comparison to propofol solved in polysorbate 80 in goats. J Vet Med A Physiol Pathol Clin Med. 2000;47:341-350.
27. Dutta S, Matsumoto Y, Ebling WF. Propofol pharmacokinetics and pharmacodynamics assessed from a cremaphor EL formulation. J Pharm Sci. 1997;86:967-969.

28. Coole A, Anderson A, Buchanan K, Byford A, et al. Water-soluble propofol analogs with intravenous anesthetic activity. Bioorg Med Chem Lett. 2001;11:927-930.

29. Trapani G, Latrofa A, Franco M, Altomare C, et al. Propofol analogues: synthesis, relationships between structure and affinity at $\mathrm{GABA}_{\mathrm{A}}$ receptor in rat brain, and differential electrophysiological profile at recombinant human GABA A receptors. J Med Chem. 1998;41:1846-1854.

30. Trapani G, Latrofa A, Franco M, Lopedota A, et al. Water-soluble salts of aminoacid esters of the anaesthetic agent propofol. Int J Pharm. 1998;175:195-204.

31. Banaszczyk M, Carlo A, Millan V, Lindsey A, et al. Propofol phosphate, a water-soluble propofol prodrug: in vivo evaluation. Anesth Analg. 2002; $95: 1285-1292$.

32. Schywalsky M, Ihmsen H, Tzabazis A, Fechner J, et al. Pharmacokinetic and pharmacodynamics of the new propofol prodrug GPI 15715 in rats. Europ J Anaesth. 2003;20:182-190.

33. Fechner J, Ihmsen H, Hatterscheid D, Schiessl C, et al. Pharmacokinetics and clinical pharmacodynamics of the new propofol prodrug GPI 15715 in volunteers. Anesthesiol. 2003;99:303-313.

34. Gibiansky E, Struys M, Gibiansky L, Vanluchene A, et al. Aquavan ${ }^{\circledR}$ injection, a water-soluble prodrug of propofol, as a bolus injection: a phase I dose-escalation comparison with Diprivan ${ }^{\circledR}$ (Part 1 - Pharmacokinetics). Anesthesiology. 2005;103:718-729.

35. Yavas S, Lizdas D, Gravenstein N, Lampotang S. Interactive web simulation for propofol and fospropofol, a new propofol prodrug. Anesth Analg. 2008;106:880-883.

36. Fechner J, Ihmsen H, Jatterscheid D, Jeleazcov C, et al. Comparative pharmacokinetics and pharmacodynamics of the new propofol prodrug GPI 15715 and propofol emulsion. Anesthesiol. 2004;101:626-639.

37. Shah A, Mistry B, Gibiansky E, Gibiansky L. Fospropofol assay issues and impact on pharmacokinetic and pharmacodynamic evaluation. Anesthesiol. 2008;109:937.

38. Klaassen C. Nonmetallic environmental toxicants. In: Hardman J, Limbird L, Gilman A, eds. The Pharmacological Basis of Therapeutics. NY: McGraw-Hill;2001:1886.

39. Ihmsen H, Tzabazis A, Schywalsky M, Schwilden H. Propofol in rats: testing for nonlinear pharmacokinetics and modeling acute tolerance to EEG effects. Eur J Anaesthesiol. 2002;19:177-188.

40. Chernik DA, Gillings D, Laine H, Hendler J, et al. Validity and reliability of the Observer's Assessment of Alertness/Sedation Scale: study with intravenous midazolam. J Clin Psychopharmacol. 1990;10:244-251.

41. Forrest F, Tooley M, Saunders P, Prys-Roberts C. Propofol infusion and the suppression of consciousness: the EEG and dose requirements. Br J Anaesth. 1994;72:35-41.

42. Kazama T, Ikeda K, Morita K, Kikura M, et al. Comparison of the effectsite $\mathrm{k}\left(\mathrm{e}_{0}\right) \mathrm{s}$ of propofol for blood pressure and EEG bispectral index in elderly and younger patients. Anesthesiol. 1999;90:1517-1527.

43. Schuttler J, Schwilden H, Stoeckel H. Infusion strategies to investigate the pharmacokinetics and pharmacodynamics of hypnotic drugs: etomidate as an example. Eur J Anaesthesiol. 1985;2:133-142.

44. Struys M, Vanluchene A, Gibiansky E, Gibiansky L, et al. Aquavan ${ }^{\circledR}$ injection, a water-soluble prodrug of propofol, as a bolus injection: a phase I dose-escalation comparison with Diprivan ${ }^{\circledR}$ (Part 2 - Pharmacodynamics and Safety). Anesthesiol. 2005;103:730-743.

45. Struys M, De Smet T, Versichelen L, Van de Velde S, et al. Comparison of closed-loop controlled administration of propofol using Bispectral Index as the controlled variable versus "standard practice" controlled administration. Anesthesiol. 2001;95:6-17.

46. Fechner J, Ihmsen H, Schiessl C, Jeleazcov C, et al. Sedation with GPI 15715, a water-soluble prodrug of propofol, using target-controlled infusion in volunteers. Anesth Analg. 2005;100:701-706.

47. Casati A, Fanelli G, Casaletti E, Colnaghi E, et al. Clinical assessment of target-controlled infusion of propofol during monitored anesthesia care. Can J Anaesth. 1999;46:235-239. 
48. Shinozaki M, Usui Y, Yamaguchi S, Okuda Y. Recovery of psychomotor function after propofol sedation is prolonged in the elderly. Can $J$ Anaesth. 2002;49:927-931.

49. Mehta A, Prakash U, Garland R, Haponik E, et al. American College of Chest Physicians and American Association for Bronchology [corrected] consensus statement: prevention of flexible bronchoscopyassociated infection. Chest. 2005;128:1742-1755.

50. Maguire G, Rubinfeld A, Trembath P, Pain C. Patients prefer sedation for fibreoptic bronchoscopy. Respirology. 1998;3:81-85.

51. Gonzalez R, De la Rosa Ramirez I, Maldonado-Hernandez A, Dominguez-Cherit G. Should patients undergoing a bronchoscopy be sedated? Acta Anaesthesiol Scand. 2003;47:411-415.

52. Colt H, Morris F. Fiberoptic bronchoscopy without premedication: a retrospective study. Chest. 1990;98:1327-1330.

53. Pickles J, Jeffrey M, Datta A, Jeffrey A. Is preparation for bronchoscopy optimal? Eur Respir J. 2003;22:203-206.

54. Silvestri G, Vincent B, Wahidi M, Robinette E, et al. A phase 3, randomized, double-blind study to assess the efficacy and safety of fospropofol disodium injection for moderate sedation in patients undergoing flexible bronchoscopy. Chest. 2009;135:41-47.

55. Greig J, Cooper S, Kasimbazi H, Monie R, et al. Sedation for fibre optic bronchoscopy. Resp Med. 1995;89:53-56.

56. Sury M, Cole P. Nalbuphine combined with midazolam for outpatient sedation: an assessment of safety in volunteers. Anaesthesia. 1988;43:281-284.

57. Bailey P, Pace N, Ashburn M, Moll J, et al. Frequent hypoxemia and apnea after sedation with midazolam and fentanyl. Anesthesiol. 1990;73:826-830.

58. Jones A, O’Driscoll R. Do all patients require supplemental oxygen during flexible bronchoscopy? Chest. 2001;119:1906-1909.

59. Vicari J. Sedation and analgesia. Gastrointest Endosc Clin N Am. 2002;12:297-311

60. Cotton P, Connor P, McGee D, Jowell P, et al. Colonoscopy: practice variation among 69 hospital-based endoscopists. Gastrointest Endosc. 2003;57:352-357.

61. Rodney W, Dabov E, Orientale E, Reeves W. Sedation associated with a more complete colonoscopy. J Fam Pract. 1993;36:394- 400.

62. Cohen L, Wecsler J, Gaetano J, Benson A, et al. Endoscopic sedation in the United States: results from a nationwide survey. Am J Gastroenterol. 2006;101:967-974.

63. Cohen L, Dubovsky A, Aisenberg J, Miller K. Propofol for endoscopic sedation: a protocol for safe and effective administration by the gastroenterologist. Gastrointest Endosc. 2003;58:725-732.

64. Heuss L, Drewe J, Schnieper P, Tapparelli C, et al. Patient-controlled versus nurse-administered sedation with propofol during colonoscopy. A prospective randomized trial. Am J Gastroenterol. 2004; 99:511-518.
65. Heuss L, Schnieper P, Drewe J, Pflimlin E, et al. Risk stratification and safe administration of propofol by registered nurses supervised by the gastroenterologist: a prospective observational study of more than 2000 cases. Gastrointest Endosc. 2003;57:664-671.

66. Rex D, Overley C, Kinser K, Coates M, et al. Safety of propofol administered by registered nurses with gastroenterologist supervision in 2000 endoscopic cases. Am J Gastroenterol. 2002;97:1159-1163.

67. AANA-ASA Joint Statement Regarding Propofol Administration. April 14, 2004.

68. Lubarsky D, Candiotti K, Harris E. Understanding modes of moderate sedation during gastrointestinal procedures: a current review of the literature. J Clin Anesth 2007;19:397-404.

69. Cohen L, Wang C, Jones J. Aquavan ${ }^{\circledR}$ is safe and effective for minimal to moderate sedation during colonoscopy. Anesthesiol 2006; 105:A1367.

70. Vargo J, Bramley T, Meyer K, Nightengale B. Practice efficiency and economics: the case for rapid recovery sedation agents for colonoscopy in a screening population. J Clin Gastroenterol. 2007;41:591-598.

71. Rex D. Review article: moderate sedation for endoscopy: sedation regiments for non-anaesthesiologists. Aliment Pharmacol Ther. 2006;24:163-171.

72. Tu R, Grewall P, Leung J, Suryaprasad A, et al. Diphenhydramine as an adjunct to sedation for colonoscopy: a double-blind randomized, placebo-controlled study. Gastrointest Endos. 2006;63:87-94.

73. Cohen L, Hightower C, Wood D, Miller K, et al. Moderate level sedation during endoscopy: a prospective study using low-dose propofol, meperidine/fentanyl, and midazolam. Gastrointest Endosc. 2004;59:795-803

74. Clarke A, Hillman L. Does the use of propofol require a specialist anesthetist? Endoscopy. 2001;33:95.

75. Van Natta M, Rex D. Propofol alone titrated to deep sedation versus propofol in combination with narcotics and/or benzodiazepines and targeted to moderate sedation for colonoscopy. Gastrointest Endosc. 2006;63:AB 192.

76. Cohen L. Clinical trial: a dose-response study of fospropofol disodium for moderate sedation during colonoscopy. Aliment Pharmacol Ther. 2008;27:597-608

77. Luer M. Phosphenytoin. Neurol Res. 1998;20:178-182.

78. http://www.news-medical.net/news/2008/12/14/44169.aspx

79. Thompson C. Fospropofol approved for monitored anesthesia care. Am J Health Syst Pharm. 2009;66:206.

80. Jantz M. The old and the new of sedation for bronchoscopy. Chest. 2009; $135: 4-6$

81. http://www.eisai.com/view_press_release.asp?ID=218\&press=206
Therapeutics and Clinical Risk Management

\section{Publish your work in this journal}

Therapeutics and Clinical Risk Management is an international, peerreviewed journal of clinical therapeutics and risk management, focusing on concise rapid reporting of clinical studies in all therapeutic areas, outcomes, safety, and programs for the effective, safe, and sustained use of medicines. This journal is indexed on PubMed Central, CAS,

\section{Dovepress}

EMBase, Scopus and the Elsevier Bibliographic databases. The manuscript management system is completely online and includes a very quick and fair peer-review system, which is all easy to use. Visit http://www.dovepress.com/testimonials.php to read real quotes from published authors. 